2010-01-01

\title{
Two-way Diffusion Model for Short Exposure Holographic Grating Formation in Acrylamide-based Photopolymer
}

Tzvetanka Babeva

Bulgarian Academy of Sciences

Izabela Naydenova

Technological University of Dublin, izabela.naydenova@tudublin.ie

Dana Mackey

Technological University of Dublin, dana.mackey@tudublin.ie

See next page for additional authors

Follow this and additional works at: https://arrow.tudublin.ie/cieoart

Part of the Condensed Matter Physics Commons, and the Optics Commons

\section{Recommended Citation}

Babeva, T. et. al. (2010) Two-way diffusion model for short-exposure holographic grating formation in acrylamidebased photopolymer. JOSA B, 27 (2), pp. 197- 203. doi:10.1364/JOSAB.27.000197

This Article is brought to you for free and open access by the Centre for Industrial and Engineering Optics at ARROW@TU Dublin. It has been accepted for inclusion in Articles by an authorized administrator of ARROW@TU Dublin. For more information, please contact arrow.admin@tudublin.ie, aisling.coyne@tudublin.ie, gerard.connolly@tudublin.ie.

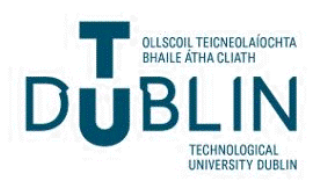




\section{Authors}

Tzvetanka Babeva, Izabela Naydenova, Dana Mackey, Suzanne Martin, and Vincent Toal

This article is available at ARROW@TU Dublin: https://arrow.tudublin.ie/cieoart/33 


\title{
Two-way diffusion model for short exposure holographic grating formation in acrylamide-based photopolymer
}

Tsvetanka Babeva ${ }^{1,2, *}$, Izabela Naydenova ${ }^{1,3}$, Dana Mackey ${ }^{1,4}$, Suzanne Martin ${ }^{1,3}$ and Vincent Toal ${ }^{1,3}$

${ }^{1}$ Centre for Industrial and Engineering Optics, Dublin Institute of Technology, Kevin Street, Dublin 8, Ireland

2 Central Laboratory of Photoprocesses, Bulgarian Academy of Sciences, Acad. G. Bonchev Str., 1113 Sofia, Bulgaria

${ }^{3}$ School of Physics, Dublin Institute of Technology, Kevin Street, Dublin 8, Ireland

${ }^{4}$ School of Mathematical Sciences, Dublin Institute of Technology, Kevin Street, Dublin 8, Ireland

*Corresponding author: babeva@clf.bas.bg

\begin{abstract}
A theoretical model for formation of a short exposure holographic grating is presented. The model accounts for both monomer and polymer diffusion and distinguishes between short polymer chains capable of diffusing and long polymer chains that are immobile. It is shown that the experimentally observed decrease of diffraction efficiency at higher spatial frequency can be predicted by assuming diffusion of short-chain polymers away
\end{abstract}


from the bright fringes. The time evolution of the refractive index modulation after short exposure is calculated and compared with experimental results. The effects of diffusion coefficients, polymerization rates, intensity and spatial frequency of recording on the properties of weak diffraction gratings are investigated by numerical simulations.

OCIS codes: $090.0090,050.2770,050.7330,160.5470,090.2900$

\subsection{Introduction}

Interest in photopolymer systems has increased markedly in the past few years. Due to their high sensitivity, self-processing and low-cost they find applications in various areas such as holography [1], manufacturing of optical elements [2,3], holographic data storage [4-6] etc. The main disadvantage of many photopolymer systems is their poor response at high-spatial-frequency recording.

Photopolymer systems usually consist of one or two monomers, an electron donor and sensitizing dye, all dispersed in a binder matrix [7]. Upon uniform illumination the monomer polymerizes and the refractive index of the system changes. When photopolymer is exposed to an interference pattern, more monomers are polymerized in the bright regions than in the dark ones. This sets up a concentration gradient of monomer which then starts to diffuse from dark to bright area where it is polymerized. The formation of surface relief grating in acrylamide based photopolymer with peaks coinciding with the areas where illumination intensity is maximum [8] as well the swelling of material in an illuminated spot observed experimentally [9] or predicted by calculations [10] can be regarded as an experimental evidence for monomer diffusion from dark to bright area. It is worth noting that if the monomer mass transfer is not 
involved in the relief formation process and the polymer shrinkage is the only mechanism involved then the peaks should appear in the nonilluminated areas. Due to polymerization and monomer diffusion a polymer density spatial distribution is formed which results in a refractive index modulation of similar form. Therefore, the recorded phase holographic grating is due to a spatial variation of refractive index resulting from changes of the density of photopolymer components.

Grating evolution in photopolymer systems has been studied theoretically and experimentally by several authors $[7,11-16]$. The common feature of the proposed models is that they fail to describe high-spatial-frequency response of photopolymers. The low diffraction efficiency at high-spatial-frequency can be explained using two approaches both referring to the non-local response of the material. This means that the response of the material at one point and time depends on what happens at other points and times in the medium. The first model, the non-local photopolymerization driven diffusion model (NPDD) $[17,18]$ assumes that the chains grow away from their initiation point resulting in "spreading" of the polymer. The model predicts that improvement at high spatial frequencies can be achieved if shorter polymer chains are created during the holographic recording [17]. Despite the successful theoretical modeling no experimental evidence for improvement of acrylamide-based photopolymer response at spatial frequency higher than 3000 lines $/ \mathrm{mm}$ has so far been achieved adopting this approach [19]. Alternatively, the two-way diffusion model [20,21], which is also based on nonlocal response of the materials, assumes that short polymer chains diffuse away from the bright fringes thus reducing the refractive index modulation. Such processes could be responsible for the decrease of diffraction efficiency at high spatial frequencies at which 
the fringe spacing is small and there is enough time for some of the polymer chains to escape from the bright fringes before the medium becoming less permeable due to complete polymerization.

To verify this assumption we propose a theoretical model for the formation of a weak grating after short exposure time. This model accounts for both monomer and polymer diffusion and moreover distinguishes between short polymer chains capable of diffusing and long polymer chains that are immobile. The time evolution of refractive index modulation after short exposure is calculated and compared with experimental results. The impact of diffusion coefficients, polymerization rates, intensity and spatial frequency of recording on the properties of weak diffraction gratings are investigated by numerical simulations.

With the present study we demonstrate that the two-way diffusion model can satisfactorily predict the poor high spatial frequency response in highly permeable photopolymers. It also predicts that the improvement of the high spatial frequency response of such systems must be directed towards decreasing the permeability of the photopolymer matrix and avoiding the creation of diffusing short polymer chains. In a more realistic picture probably both non-local processes - non-local polymerization and short polymer diffusion take place and should be taken into account in order to achieve the ultimate high spatial frequency response.

\subsection{Two-way diffusion model}

As stated above the variation of monomer concentration (in time and space) during illumination is due to the monomer polymerization and monomer diffusion. Generally, 
these two processes are expressed mathematically using a standard one-dimensional diffusion equation [7]:

$$
\frac{\partial m(x, t)}{\partial t}=\frac{\partial}{\partial x}\left[D_{m}(x, t) \frac{\partial m(x, t)}{\partial x}\right]-F(x, t) m(x, t),
$$

where $m(x, t)$ is monomer concentration, $F(x, t)$ is polymerization rate and $D_{m}(x, t)$ is monomer diffusion coefficient. The polymerization rate depends on free radical concentration that is a function of radicals generation and termination rates. For constant intensity and for the short exposure time of 0.1-0.3 s used in our studies we can assume that the rate of free radicals generation is constant because there are plenty of unbleached dye molecules available to absorb and generate radicals. Furthermore, due to the insignificant changes in material's viscosity for such a short exposure time we can assume that the termination rate is also constant (i.e the Trommsdorff effect can be ruled out). Therefore in the case of short exposure time we can assume the polymerization rate not to change in time. Further we suppose that the polymerization rate is proportional to the intensity of illumination:

$$
F(x)=k_{p} I^{a}(x)=k_{p} I_{0}^{a}[1+V \cos (K x)]^{a}=F_{0}[1+V \cos (K x)]^{a} \equiv F_{0} f(x),
$$

where $I(x)=I_{0}[1+V \cos (K x)]$ is the illumination pattern intensity, $I_{0}$ is the average intensity, $V=2 \sqrt{I_{1} I_{2}} /\left(I_{1}+I_{2}\right)$ is the fringe visibility, $I_{1}$ and $I_{2}$ the intensities of the writing beams, $K=2 \pi / \Lambda$ the grating vector, $\Lambda$ the grating period and $F_{0}=k_{p} I_{0}^{a}$, where 
$k_{p}=0.1 \mathrm{~s}^{-1}\left(\mathrm{~mW} / \mathrm{cm}^{2}\right)^{-\mathrm{a}}$ is a fixed constant [22]. For recording intensities from 1 to 100 $\mathrm{mW} / \mathrm{cm}^{2}$ and $a=0.3-0.5$ the polymerization time is between 10 and $1 \mathrm{~s}$. Thus, for short exposure times $(0.1-0.2 \mathrm{~s})$ the changes in the permeability of the medium are insignificant and one can assume that $D_{m}$ is constant in time. Concerning the spatial variation of $D_{m}$ it was shown that even the first order term in Fourier series expansion of $D_{m}$ has a rather small effect [7], so we can assume that $D_{m}$ is constant. With these assumptions eq. 1, describing the rate of change of monomer concentration, takes the simpler form:

$$
\frac{\partial m(x, t)}{\partial t}=D_{m} \frac{\partial^{2} m(x, t)}{\partial x^{2}}-\Phi(t) F(x) m(x, t),
$$

where we introduce the step function $\Phi(t)$ to account for the short exposure regime with $t_{e}$ being the exposure time:

$$
\Phi(t)=\left\{\begin{array}{lll}
1, & \text { if } & t \leq t_{e} \\
0 & \text { if } & t>t_{e}
\end{array}\right.
$$

As could be seen from eqs. 3 and 4, the proposed model assumes that the polymerization stops as soon as the illumination is turned off. This is a simplification that can be justified by the fact that experimentally we observe little or no change in diffraction efficiency following termination of longer exposures. Further, in our model we distinguish two types of polymer chains: short chains, $p_{1}$, capable of diffusing and long chains, $p_{2}$, that are immobile. We assume that short chains are converted to long chains at a rate proportional to monomer and short polymer concentrations and introduce a parameter $\Gamma$, 
which is the conversion rate constant. Then the equations for temporal and spatial evolution of $p_{1}$ and $p_{2}$ take the form:

$$
\begin{aligned}
& \frac{\partial p_{1}(x, t)}{\partial t}=\frac{\partial}{\partial x}\left[D_{p}(x) \frac{\partial p_{1}(x, t)}{\partial x}\right]+\Phi(t)\left[F(x) m(x, t)-\Gamma m(x, t) p_{1}(x, t)\right] \\
& \frac{\partial p_{2}(x, t)}{\partial t}=\Phi(t) \Gamma m(x, t) p_{1}(x, t)
\end{aligned}
$$

where $D_{p}$ is the polymer diffusion coefficient. Further we assume that $D_{p}$ is proportional to the interference pattern, which means that the maximum values of $D_{p}$ coincide with the peaks in intensity. From the other hand it is known that at higher intensity more shortchain polymer molecules are formed [23]. Considering that the centre of the bright fringes will be rich of short polymers and the edges will be poor, the assumption in Eq. 6 means that the diffusion coefficient for shorter chains will be higher than the diffusion coefficient for longer chains

$$
D_{p}(x)=D_{p}(1+V \cos (K x))^{a} \equiv D_{p} f(x)
$$

In this simplified picture we also assume, as it is seen from eq 5 that the conversion from short to long polymer chains also stop when the exposure is stopped. For the purpose of the subsequent analysis and numerical simulations, we introduce dimensionless variables

$$
\bar{x}=\frac{x}{\Lambda}, \quad \bar{t}=\frac{t}{t_{0}}, \quad \bar{m}=\frac{m}{m_{0}}, \quad \bar{p}_{i}=\frac{p_{i}}{m_{0}}(i,=1,2)
$$

where $m_{0}=m(x, 0)$ is the initial monomer concentration and $t_{0}=1 \mathrm{~s}$. The value of $t_{0}$ is chosen to be $1 \mathrm{~s}$ for two reasons. The first one is for convenience. When $t_{0}$ is $1 \mathrm{~s}$ the 
dimensionless time used in computations will be the same as the real time (that is in seconds). For values of $t_{0}$ different from $1 \mathrm{~s}$ a correction factor will be needed to transform the dimensionless time to the real time. The second reason is that when nondimensionalising a system of physical equations it is customary to scale variables by values with a similar order of magnitude. As the exposure time is $0.2-0.3 \mathrm{~s}$ and the total simulation times did not exceed $10 \mathrm{~s}$, we considered $t_{0}=1 \mathrm{~s}$ as a good reference time.

It is also common practice to choose scales which have special significance in the physical problem such as, for example, diffusion or polymerization time. However, we avoided such scales here as we varied these parameters, which affect the diffusion and polymerization rates and consequently would distort the time and dynamics of the whole problem. The model equations become

$$
\begin{aligned}
& \frac{\partial \bar{m}}{\partial \bar{t}}=\kappa t_{0} \frac{\partial^{2} \bar{m}}{\partial \bar{x}^{2}}-\Phi(\bar{t}) F_{0} t_{0} f(\bar{x}) \bar{m} \\
& \frac{\partial \bar{p}_{1}}{\partial \bar{t}}=\kappa \varepsilon t_{0} \frac{\partial}{\partial \bar{x}}\left[f(\bar{x}) \frac{\partial \bar{p}_{1}}{\partial \bar{x}}\right]+\Phi(\bar{t})\left[F_{0} t_{0} f(\bar{x}) \bar{m}-\gamma \overline{m p}_{1}\right] \\
& \frac{\partial \bar{p}_{2}}{\partial \bar{t}}=\Phi(\bar{t}) \gamma \overline{m p} \bar{p}_{1}
\end{aligned}
$$

where $\kappa=D_{m} / \Lambda^{2}, \varepsilon=D_{p} / D_{m}$ and $\gamma=m_{0} t_{0} \Gamma$. The non-dimensional initial and boundary conditions are:

$$
\bar{m}(\bar{x}, 0)=1, \quad \bar{p}_{i}(\bar{x}, 0)=0, \quad \frac{\partial \bar{m}}{\partial \bar{x}}(\bar{x}, \bar{t})=\frac{\partial \bar{p}_{1}}{\partial \bar{x}}(\bar{x}, \bar{t})=\frac{\partial \bar{p}_{2}}{\partial \bar{x}}(\bar{x}, \bar{t})=0, \text { for } \bar{x}=0,1
$$


We have imposed zero-flux boundary conditions as we expect the final monomer and polymer concentration patterns to exhibit minima or maxima at the ends of the interval $[0, \Lambda]$, which are maximum points for the illumination intensity. It should be noted that by integrating the eqs 8 one can obtain the conservation law that is expected because the total concentration of different phases (monomer, short and long polymers) remains constant:

$$
\int_{0}^{1}\left[\bar{m}(\bar{x}, \bar{t})+\bar{p}_{1}(\bar{x}, \bar{t})+\bar{p}_{2}(\bar{x}, \bar{t})\right] d \bar{x}=1
$$

\subsection{Calculation of refractive index modulation}

As explained in the previous paragraph, a polymer density spatial modulation is formed upon illumination which results in refractive index modulation with a similar pattern to that of the illumination. The refractive index modulation is the difference between the refractive indices in the illuminated and in non-illuminated areas. If we consider both areas as effective mixtures of monomer $(m)$, short $\left(p_{1}\right)$ and long $\left(p_{2}\right)$ chain polymer molecules and a binder $(b)$ their refractive indices could be expressed using Lorentz-Lorenz equation in the form [24]:

$$
\begin{gathered}
\frac{n_{e}^{2}-1}{n_{e}^{2}+2}=\varphi_{m} \frac{n_{m}^{2}-1}{n_{m}^{2}+2}+\varphi_{p 1} \frac{n_{p 1}^{2}-1}{n_{p 1}^{2}+2}+\varphi_{p 2} \frac{n_{p 2}^{2}-1}{n_{p 2}^{2}+2}+\varphi_{b} \frac{n_{b}^{2}-1}{n_{b}^{2}+2}, \\
\varphi_{b}+\varphi_{m}+\varphi_{p 1}+\varphi_{p 2}=1
\end{gathered}
$$

where $n_{e}$ is the effective refractive index of the mixture; $\varphi_{m}, \varphi_{p 1}, \varphi_{p 2}$ and $\varphi_{b}$ are the volume fractions of components $\left(\varphi_{i=} V_{i} / V_{\text {tot }}\right.$, where $V_{i}$ and $V_{\text {tot }}$ are the volume occupied by the $i$-th 
component and the total volume, respectively) and $n_{m}, n_{p 1}, n_{p 2}$ and $n_{b}$ are the refractive indices of the components. In our numerical simulations we used the values of the refractive index of each component that was determined previously from spectrophotometric measurements $\left(n_{m}=1.55, n_{p 1}=n_{p 2}=1.64\right.$ and $n_{b}=1.496$ at $\left.532 \mathrm{~nm}\right)$.

Further using the normalized concentrations of the components calculated from the model $\left(\bar{m}, \bar{p}_{1}\right.$ and $\left.\bar{p}_{2}\right)$ and considering the densities for all components we calculated the volume fraction of each component:

$$
\begin{aligned}
& \varphi_{m}=\frac{\bar{m} / \rho_{m}}{\left(b / m_{0}\right)\left(\rho_{b} / \rho_{m}\right)+\left(\bar{m} / \rho_{m}\right)+\left(\bar{p}_{1}+\bar{p}_{2}\right) / \rho_{p}} \\
& \varphi_{p 1}=\frac{\bar{p}_{1} / \rho_{p}}{\left(b / m_{0}\right)\left(\rho_{b} / \rho_{m}\right)+\left(\bar{m} / \rho_{m}\right)+\left(\bar{p}_{1}+\bar{p}_{2}\right) / \rho_{p}} \\
& \varphi_{p 2}=\frac{\bar{p}_{2} / \rho_{p}}{\left(b / m_{0}\right)\left(\rho_{m} / \rho_{b}\right)+\left(\bar{m} / \rho_{m}\right)+\left(\bar{p}_{1}+\bar{p}_{2}\right) / \rho_{p}},
\end{aligned}
$$

In Eq $12 m_{0}$ is the initial monomer concentration and $\rho_{i}(i=m, b, p)$ are the densities of the components. They are equal to $1.3 \mathrm{~g} / \mathrm{cm}^{3}$ for polymer [19], $1.15 \mathrm{~g} / \mathrm{cm}^{3}$ for monomer and $1.19 \mathrm{~g} / \mathrm{cm}^{3}$ for binder. The values for monomer and binder densities are obtained considering the masses of the components (see Sec. 4.1) and their densities [19]. To make the picture more realistic, in the denominator of eq. 12 which is the total volume of the sample, we introduce the parameter $b / m_{0}$ as the ratio of the masses of the binder and monomer. In this way we account both for the presence of binder and for the fact that monomer occupies about $17 \%$ of the total volume.

The temporal changes of the volume fractions of all components $\left(\varphi_{m}, \varphi_{p 1}, \varphi_{p 2}\right.$ and $\left.\varphi_{b}\right)$ can be estimated from Eq. 12 where the variations of $\bar{m}, \bar{p}_{1}$ and $\bar{p}_{2}$ in time are 
calculated by the model equations (Eqs. 8 and 9). Further $\varphi_{m}, \varphi_{p 1}, \varphi_{p 2}$ and $\varphi_{b}\left(=1-\varphi_{m}+\varphi_{p 1}\right.$ $\left.+\varphi_{p 2}\right)$ are used in Eq.11 for calculation of effective refractive index as a function of time. The temporal growth of refractive index modulation that gives rise to the first order of diffraction was then calculated as:

$$
\Delta n=n_{\text {emax }}(t)-n_{\text {emin }}(t), .
$$

where $n_{\text {emax }}(t)$ and $n_{\text {emin }}(t)$ are effective refractive index in the centres of the bright and dark fringes, respectively.

\subsection{Numerical simulations}

The non-dimensional model equations were integrated numerically using a standard Crank-Nicolson finite difference method [25]. The numerical value for the monomer diffusion coefficient $\left(D_{m}=1.3 \times 10^{-8} \mathrm{~cm}^{2} / \mathrm{s}\right)$ was taken from the experimental data previously published in [16] and the ratio $\varepsilon$ between polymer and monomer diffusion coefficient was varied between $0.001-0.1$. The influence of the polymerization rate $F_{0}$ was studied for values of $0.1,0.3$ and $1 \mathrm{~s}^{-1}$. The spatial frequencies of recording were varied from 200 to $5000 \mathrm{l} / \mathrm{mm}$, which covered grating periods from 5 to $0.2 \mu \mathrm{m}$ respectively. The proportionality constant values between recording intensity and polymerization rate are $a=1[7,13], 0.5$ [12] or 0.3 [22]. It may not be straightforward to determine experimentally the rate of conversion of short to long polymer chains $\gamma$, that is why it was varied between 0 and 100 . The exposure time was $0.2 \mathrm{~s}$ unless otherwise specified. 
Some concentration profiles of monomer and polymers calculated from eqs. 8 are shown in Figs. 1 and 2 for low and high spatial frequencies, respectively.

All components - monomer, short and long polymers developed spatial modulation with concentration minimum for monomer and maximum for polymer in the centre of the bright fringes $(x=0,1,2)$. Because the exposure time $(0.2 \mathrm{~s})$ is small compared to the polymerization time $(3.3 \mathrm{~s})$ most of the monomer (more than $95 \%$ ) remains unpolymerized after such a short period of initial illumination. From Fig. 2 it can be seen that the concentration profile of monomer is almost flat for higher spatial frequencies. Because of the small fringe spacing $\left(200 \mathrm{~nm}\right.$ ), the monomer needs less time (about $8 \times 10^{-4}$ s) to diffuse from dark to bright fringes and to reestablish the concentration equilibrium disturbed by the decreased number of monomer molecules due to their participation in the photopolymerization process. As a result, the spatial modulation of monomer disappears very quickly. Similarly equalizing the monomer concentration in space takes place for low spatial frequency recording but this process is slower compared to the highfrequency case. The monomer diffusion time at $5001 / \mathrm{mm}(5 \mathrm{~m})$ is about $0.08 \mathrm{~s}$ and can be observed with the present experimental arrangement.

A comparison of Figs. $1 \mathrm{~b}$ and $2 \mathrm{~b}$ shows that the spreading of the polymer out from bright fringes is more pronounced for high spatial frequencies at which the distances and diffusion times are smaller. For $5000 \mathrm{l} / \mathrm{mm}$ the widening of illuminated area is faster than at $500 \mathrm{1} / \mathrm{mm}$ where more time is needed for diffusion of short polymer chains away from bright fringes. It is worth noting the different time scales for Figs. $1 \mathrm{~b}$ and $2 \mathrm{~b}$. The concentration profiles for long-chain polymer molecules (Figs. 1c and 2c) do not change after exposure and neither is further widening of illuminated area observed with time. 
The reason for that is that long polymer chains are assumed to be immobile and, once formed at a particular location, cannot move to another.

Fig. 3 presents the evolution of the calculated refractive index modulation with time at low $(500 \mathrm{l} / \mathrm{mm})$ and high $(5000 \mathrm{l} / \mathrm{mm})$ spatial frequency of recording for three different ratios of polymer and monomer diffusion coefficients. It is seen that, after the illumination is stopped, refractive index modulation decreases more rapidly at both higher spatial frequencies and higher $D_{p}$. Considering that this decrease is due to diffusion of short polymer chains away from the bright fringes it can be expected that the decrease will be more rapid for higher values of $D_{p}$ as well as for higher spatial frequencies where the fringe spacing is smaller.

The influence of the polymerization rate on the post-exposure dynamics of refractive index modulation at low $(5001 / \mathrm{mm})$ and high $(5000 \mathrm{l} / \mathrm{mm})$ spatial frequencies can be seen from Fig. 4. Considering that $F_{0}$ is proportional to intensity of illumination (see eq. 2) the dependences in Fig. 4 can be also regarded as intensity dependences of refractive index modulation. It is seen that as $F_{0}$ decreases the refractive index modulation also decreases. The reason for this is that at low $F_{0}$ less monomer is converted to polymer during the illumination. It is seen that, after illumination ceases, $\Delta n$ decreases more rapidly for higher values of $F_{0}$ (i.e higher intensity). This can be explained by the fact that at higher intensity more short chain polymers are formed [23]. They are mobile and can easily escape from bright fringe regions resulting in a decrease of $\Delta n$.

The results presented in Figs. 3 and 4 show that the model predicts the drop in refractive index modulation at higher spatial frequency which is experimentally observed. This will be discussed in more detail in the next section. 
Fig.5 presents the influence of rate of conversion $\gamma$ from short to long polymer chains on the refractive index modulation for weak gratings with spatial frequencies of $2000 \mathrm{l} / \mathrm{mm}$. Small values of $\gamma$ mean that the conversion from short to long chains is slow, so polymer molecules are mobile for longer times and can diffuse away from bright fringes reducing the refractive index modulation. On the other hand higher values of $\gamma$ mean that short chains are converted to long chains faster leading to slow decrease of refractive index modulation due to the fact that long polymer chains are incapable of diffusing away from bright fringes. As expected it is seen from Fig. 5 that the refractive index modulation decreases very rapidly when $\gamma$ is small. On the other hand, for high values of $\gamma$ the decrease in $\Delta n$ is slower.

From the numerical simulations presented in Fig. 3(b) and Fig. 5 it can be seen that the high spatial frequency response could be improved by suppressing the diffusion of short polymer chains and by choosing the recording parameters so as to favor the rapid conversion of short to long-chain polymers. Following this strategy and choosing a binder with low permeability we have already been successful in recording reflection holograms in acrylamide-based photopolymer [26, 27].

\subsection{Experimental data}

\subsection{Materials}

The photosensitive layers were prepared by adding $2 \mathrm{ml}$ of triethanolamine, $0.6 \mathrm{~g}$ acrylamide, $0.2 \mathrm{~g} \mathrm{~N}, \mathrm{~N}$-methylene bisacrylamide and $4 \mathrm{ml}$ Erythrosin B dye of $1.1 \mathrm{mM}$ dye stock solution to $17.5 \mathrm{ml}$ stock solution of polyvinilalcohol $(10 \mathrm{w} / \mathrm{w})$ [28]. Amounts of 2 $\mathrm{ml}$ of the well mixed solution were gravity settled on levelled glass substrates so that the 
upper sides of the layers were open to the air. The thickness of the layers after drying for $24 \mathrm{~h}$ in darkness under normal laboratory conditions $\left(t^{\circ}=(21-23){ }^{\circ} \mathrm{C}\right.$ and $\mathrm{RH}=(40-60)$ \%) was $150 \pm 3 \mu \mathrm{m}$.

\subsection{Recording of gratings}

Transmission gratings with spatial frequency in the range $200-3000 \mathrm{l} / \mathrm{mm}$ and diffraction efficiency of a few percent were recorded using $\mathrm{NdYVO}_{4}$ laser (Verdi 05) $($. . $532 \mathrm{~nm})$ using short exposure times $(0.2-2 \mathrm{~s})$. A He-Ne laser, ( . $633 \mathrm{~nm})$ was used for monitoring the real-time evolution of diffraction efficiency. The refractive index modulation was calculated from the measured diffraction efficiency using Kogelnik's coupled wave theory [29].

\subsection{Results and discussions}

In this section we illustrate with two examples the good agreement that has been obtained between the results obtained from gratings and those obtained from the model presented here for refractive index modulation of weak gratings.

Fig. 6 presents the comparison between modeled and measured curves of refractive index modulation in the case of weak gratings at different spatial frequencies between 200 and $3000 \mathrm{l} / \mathrm{mm}$ and different intensities values (see the captions of Fig. 6).

It is seen that the model predicts qualitatively very well the behavior of the refractive index modulation. For the same intensity (i.e $F_{0}$ ) the initial slope of the graph of $\Delta n$ versus exposure time is the same for gratings with spatial frequency of 200, 500 and 1000 $1 / \mathrm{mm}$. With decreasing intensity, the slope also decreases. Further with increasing spatial 
frequency the amplitude of refractive index modulation decreases. Additionally the model predicts very well the post-exposure increase of $\Delta n$ for low spatial frequency $(200 \mathrm{l} / \mathrm{mm})$ [20]. It can be seen from Fig. 6 that the values of $\Delta n$ predicted by the model are higher than the measured ones. This difference may be due to the discrepancies between the real values of refractive indices of the photopolymer components and the values assumed in the model. From eqs. 11 and 13 it is seen that the calculated $\Delta n$ is a function of the monomer, polymer and binder refractive index as well as their volume fractions. It is relatively easy to determine the monomer and the binder refractive indices. (For example transmittance and reflectance measurements on the respective layers [30]). However the determination of polymer refractive index is not so straightforward because it depends on the degree of polymerization. In our simulations we used $n_{p}$ value that is determined from spectrophotometric measurements of a bulk polymerized layer, i.e one that is uniformly illuminated. However under conditions of spatially non-uniform polymerization it may happen that the degree of polymerization is different. Moreover, at short illumination time it is possible that the monomers are not fully polymerized as it is in the case of bulk polymerization and refractive index of the polymer fraction is different than 1.64 (the refractive index of uniformly polymerized material). Our additional simulations have shown that if we decrease the value of np from 1.64 to keeping all parameters the same refractive index modulation decreases ...

Fig. 7 presents the comparison between simulated and measured refractive index modulation in the case of constant exposure of $7 \mathrm{~mJ} / \mathrm{cm}^{2}$ for a short exposure grating of spatial frequency of $500 \mathrm{l} / \mathrm{mm}$. We obtained constant recording exposure of $7 \mathrm{~mJ} / \mathrm{cm}^{2}$ using exposure times of 2, 0.5 and $0.1 \mathrm{~s}$ and recording intensities of 3.5, 14 and 70 
$\mathrm{mW} / \mathrm{cm}^{2}$, respectively). The good agreement regarding the shapes and slopes of the curves can be easily seen, but the calculated values of $n$ are again higher than the measured ones.

\section{Conclusion}

A two-way diffusion model for short exposure holographic grating formation in acrylamide-based photopolymer is presented. Accounting for both monomer and polymer diffusion the model predicts the experimentally observed drop in refractive index modulation at high spatial frequency. Moreover, the model distinguishes between short polymer chains capable of diffusing and long polymer chains that are immobile.

The numerical simulations show that the suppression of short polymer diffusion improves the high spatial frequency response and that fast conversion of short to long polymer chains has a positive effect on the final refractive index modulation. Further, higher recording intensities generate larger numbers of short polymer chains leading to higher post-exposure reduction in refractive index modulation. Therefore low intensity recording is more appropriate for high-spatial frequency recording. Following this strategy and choosing a binder with low permeability improved the spatial frequency response and we have already been successful in recording reflection holograms in acrylamide-based photopolymer [26,27].

It was demonstrated that a good agreement between the theoretically predicted and the experimentally measured refractive index modulation curves can be obtained using the two-way diffusion model. 


\section{Acknowledgement}

This publication has resulted from research conducted with the financial support of

Science Foundation Ireland grant No 065/RFP/PHY085 and COST Action MP0604.

The authors would like to acknowledge the School of Physics at DIT and Facility for Optical characterization and Spectroscopy, DIT, for technical support.

T. Babeva would like to thank the Arnold F Graves Postdoctoral programme at DIT.

\section{References}

1. T. J. Trout, J. J. Schmieg, W. Y. Gambogi, and A. M. Weber, "Optical photopolymers: Design and applications," Adv. Mat. 10, 1219-1224 (1998).

2. A. Sullivan, M. Grabowski, and R. McLeod, "Three-dimensional direct-write lithography into photopolymer," Appl. Opt. 46, 295-301 (2007).

3. S. Guntaka, V. Toal, and S. Martin, "Holographically recorded photopolymer diffractive optical element for holographic and electronic speckle-pattern Interferometry,” Appl. Opt. 41, 7475-7479 (2002).

4. H. Sherif, I, Naydenova, S. Martin, C. McGinn, and V. Toal, "Characterization of an acrylamide-based photopolymer for data storage utilizing holographic angular multiplexing,” J. Opt. A:Pure and Appl. Opt. 7, 255-261 (2005).

5. http://www.inphase-technologies.com/

6. http://www.aprilisinc.com/ 
7. G. Zhao and P. Mouroulis, "Diffusion model of hologram formation in dry photopolymer materials," J. Mod. Opt. 41, 1929-1939 (1994).

8. I. Naydenova, E. Mihaylova, S. Martin, and V. Toal, "Holographic patterning of acrylamide-based photopolymer surface,” Opt. Express 13, 4878-4889 (2005).

9. Tz. Babeva, I. Naydenova, S. Martin and V. Toal, "Method for real-time characterization of diffusion properties of polymerisable systems", Opt. Express 16, 8487-8497, (2008).

10. S. Gallego, A. Márquez, S. Marini, E. Fernández, M. Ortuño, I. Pascual, "In dark analysis of PVA/AA materials at very low spatial frequencies: phase modulation evolution and diffusion estimation", Opt. Express 17, 18279-18291, (2009).

11. S. Piazzola and B. Jenkins, "First-harmonic diffusion model for holographic grating formation in photopolymers", J. Opt. Soc. Am. B 17, 1147-1157 (2000).

12. V. Moreau, Y. Renotte, and Y. Lion, "Characterization of DuPont photopolymer: determination of kinetic parameters in a diffusion model," Appl. Opt. 41, 3427-3435 (2002).

13. V. L. Colvin, R. G. Larson, A. L. Harris, and M. L. Schilling, "Quantitative model of volume hologram formation in photopolymers," J. Appl. Phys. 81, 5913-5923 (1997).

14 S. Blaya, L. Carretero, R. Mallavia, A. Fimia, and R. F. Madrigal, "Holography as a technique for the study of photopolymerization kinetics in dry polymeric films with a nonlinear response”, Appl. Opt. 38, 955-962 (1999).

15. J. H. Kwon, H. C. Hwang, and K. C. Woo, "Analysis of temporal behavior of beams diffracted by volume gratings formed in photopolymers", J. Opt. Soc. Am. B 16, 1651-1657 (1999). 
16. C. Neipp, S. Gallego, M. Ortuno, A. Marquez, M. L. Alvarez, A. Belendez and I. Pascual, "First-harmonic diffusion-based model applied to a polyvinyl-alcoholacrylamide-based photopolymer”, J. Opt. Soc. Am. B 20, 2052-2060 (2003).

17. J. T. Sheridan and J. R. Lawrence, "Nonlocal-response diffusion model of holographic recording in photopolymer”, J. Opt. Soc. Am. A 17, 1108-1114 (2000).

18. J. T. Sheridan, M. Downey, F. T. O’Neill, "Diffusion based model of holographic grating formation in photopolymers: Generalised non-local material responses," J. Opt. A: Pure and Appl. Opt. 3, 477-488 (2001).

19. M. R. Gleeson, D. Sabol, S. Liu, C. E. Close, J. V. Kelly, and J. T. Sheridan, "Improvement of the spatial frequency response of photopolymer materials by modifying polymer chain length”, J. Opt. Soc. Am. B 25, 396-406 (2008).

20. I. Naydenova, R. Jallapuram, R. Howard, S. Martin, and V. Toal, "Investigation of the Diffusion Processes in a Self-Processing Acrylamide-Based Photopolymer System,” Appl. Opt. 43, 2900-2905 (2004).

21. S. Martin, I. Naydenova, R. Jallapuram, R. Howard, and V. Toal, “Two-way diffusion model for the recording mechanism in a self developing dry acrylamide photopolymer," Proc. SPIE 6252, 62525-625217 (2006).

22. R. Jallapuram, I. Naydenova, H. J. Byrne, S. Martin, R. Howard and V. Toal, "Raman spectroscopy for the characterization of the polymerization rate in an acrylamidebased photopolymer," Appl. Opt. 47, 206-212 (2008).

23. P. Munk and T. M. Aminabhavi, Introduction to macromolecular science (Jonh Wiley \& Sons, Inc., New York, 2002). 
24. I. Aubrecht, M. Miler and I. Koudela, "Recording of holographic diffraction gratings in photopolymers: theoretical modelling and real-time monitoring of grating growth" J. Modern Opt. 45, 1465-1477 (1998).

25. C. Grossman, H-G. Roos, M. Stynes, Numerical Treatment of Partial Differential Equations (Springer, 2007).

26. I. Naydenova, R. Jallapuram, V. Toal, and S. Martin, "A visual indication of environmental humidity using a color changing hologram recorded in a selfdeveloping photopolymer", Appl. Phys Letters 92, 031109 (2008).

27. I. Naydenova, H. Sherif, S. Martin, R. Jallapuram, and V. Toal, Patent No. WO2007060648 (2007).

28. S. Martin, C. A. Feely, and V. Toal, "Holographic recording characteristics of an acrylamide-based photopolymer,” Appl. Opt. 36, 5757-5768 (1997).

29. H. Kogelnik, "Coupled wave theory for thick hologram gratings", Bell Syst. Tech. J. 48, 2909-2947 (1969).

30. T. Babeva, R. Todorov, S. Mintova, T. Yovcheva, I. Naydenova and V Toal, "Optical properties of silica-MFI doped acrylamide-based photopolymer", J. Opt. A: Pure and Appl. Opt. 11, 024015, (2009). 


\section{Captions to the figures}

Fig. 1 (Color online) Numerical results for the concentration profiles of monomer - (a), short- (b) and long- (c) chain polymers for spatial frequency of 500 lines/mm (exposure time is $0.2 \mathrm{~s}, F_{0}=0.3 \mathrm{~s}^{-1}, a=0.5, \gamma=1, D_{p} / D_{m}=0.01$ ).

Fig. 2 (Color online) Numerical results for the concentration profiles of monomer- (a), short- (b) and long- (c) chain polymers for spatial frequency of 5000 lines/mm (exposure time is $0.2 \mathrm{~s}, F_{0}=0.3 \mathrm{~s}^{-1}, a=0.5, \gamma=1, D_{p} / D_{m}=0.01$ ).

Fig. 3 (Color online) Time evolution of refractive index modulation for weak gratings with spatial frequency of $500 \mathrm{1} / \mathrm{mm}$ (a) and $5000 \mathrm{1} / \mathrm{mm}$ (b) at different ratios $D_{p} / D_{m}\left(F_{0}=0.3 \mathrm{~s}^{-1}, a=0.5, \gamma=1\right)$ (the dotted vertical line shows the time when light is turned off)

Fig. 4 (Color online) Time evolution of refractive index modulation for weak gratings with spatial frequency of $500 \mathrm{1} / \mathrm{mm}$ (a) and $5000 \mathrm{1} / \mathrm{mm}$ (b) at different polymerization rates $\left(D_{p} / D_{m}=0.01, a=0.5, \gamma=1\right)$ (the dotted vertical line shows the time when light is turned off)

Fig. 5 (Color online) Time evolution of refractive index modulation for weak gratings with spatial frequency of $2000 \mathrm{l} / \mathrm{mm}$ at different rates of conversion from short to long polymer chains $\left(D_{p} / D_{m}=0.01, a=0.5, F_{0}=0.3 \mathrm{~s}^{-1}\right)$ (the dotted vertical line shows the time when light is turned off)

Fig. 6 (Color online) Numerically simulated (a) and experimentally measured (b) refractive index modulation for weak gratings at different spatial frequencies $\left(t_{\mathrm{exp}}=0.3 \mathrm{~s}, a=0.5, F_{0}=0.15 \mathrm{~s}^{-1}\right.$ for 200,500 and $1000 \mathrm{l} / \mathrm{mm}, F_{0}=0.10$ and $0.05 \mathrm{~s}^{-1}$ 
for 2000 and $3000 \mathrm{l} / \mathrm{mm}, D_{p} / D_{m}=0.01, \gamma=10$ ) (the dashed vertical line shows the time when light is turned off)

Fig. 7 (Color online) Numerically simulated (a) and experimentally measured (b) refractive index modulation for weak gratings $5001 / \mathrm{mm}$ at exposure of 7 $\mathrm{mJ} / \mathrm{cm}^{2}$ ( $t_{e}$-recording time, $I$ - intensity) $\left(a=0.5, D_{p} / D_{m}=0.01, \gamma=1, F_{0}=0.84\right.$, 0.37 and $0.19 \mathrm{~s}^{-1}$ for $t_{e}=0.1,0.5$ and $2 \mathrm{~s}$ ) (the dashed vertical lines show the time when light is turned off) 


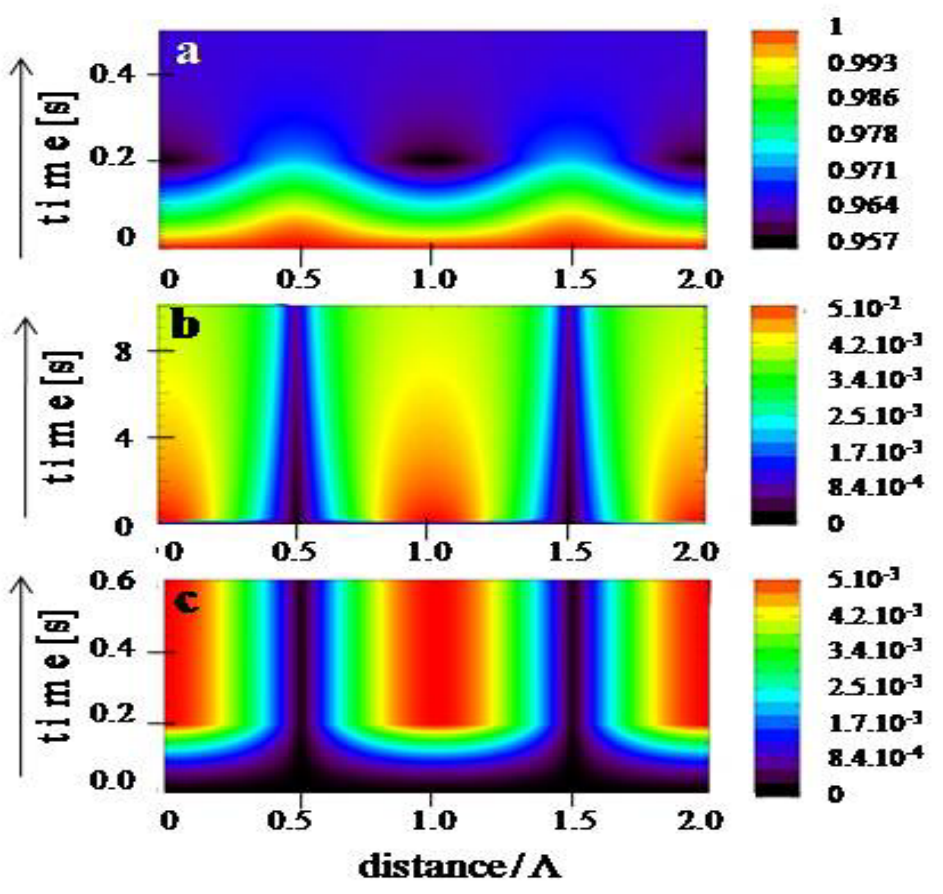

Figure 1 


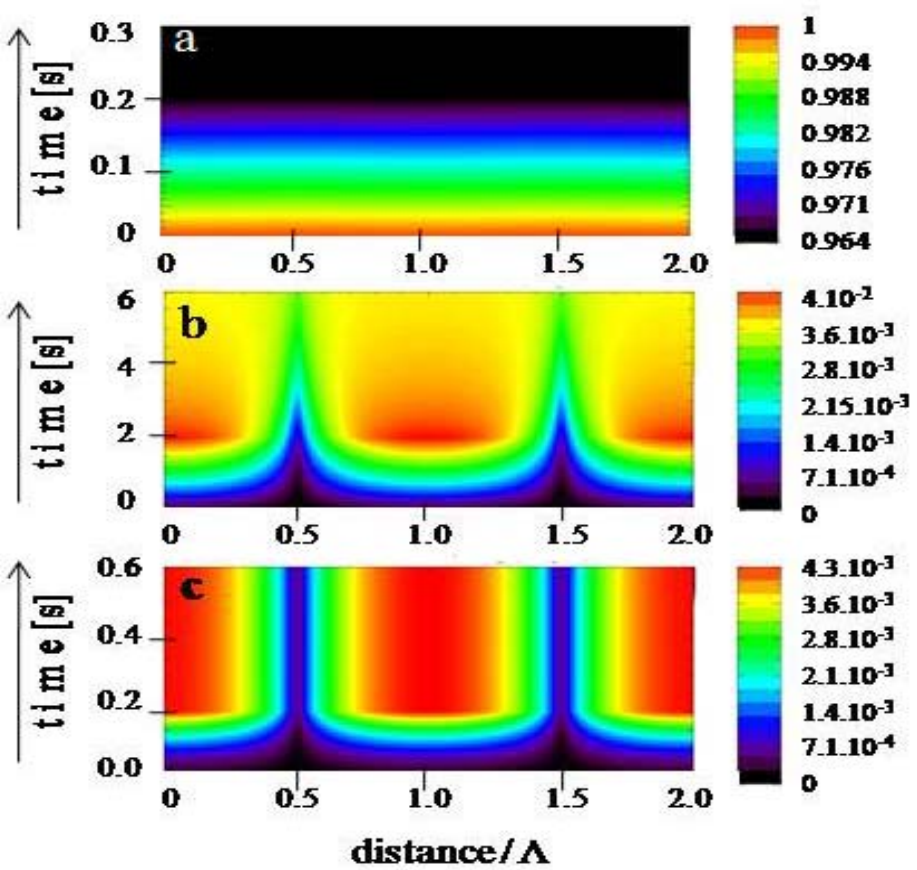

Figure 2 

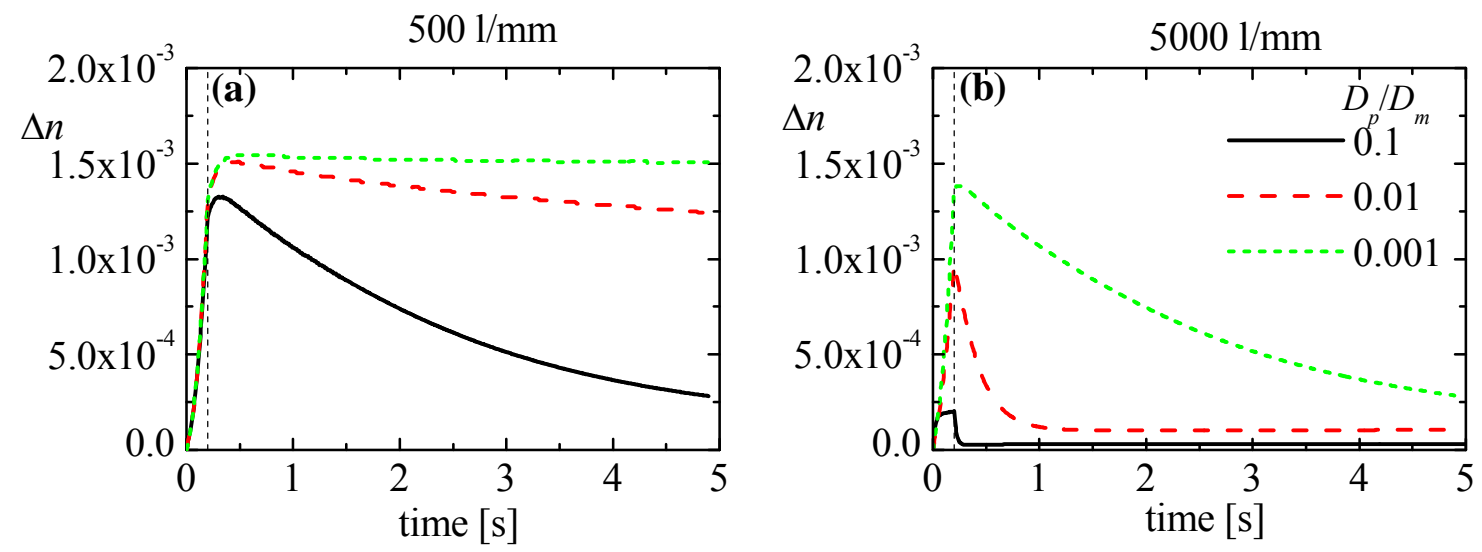

Figure 3 

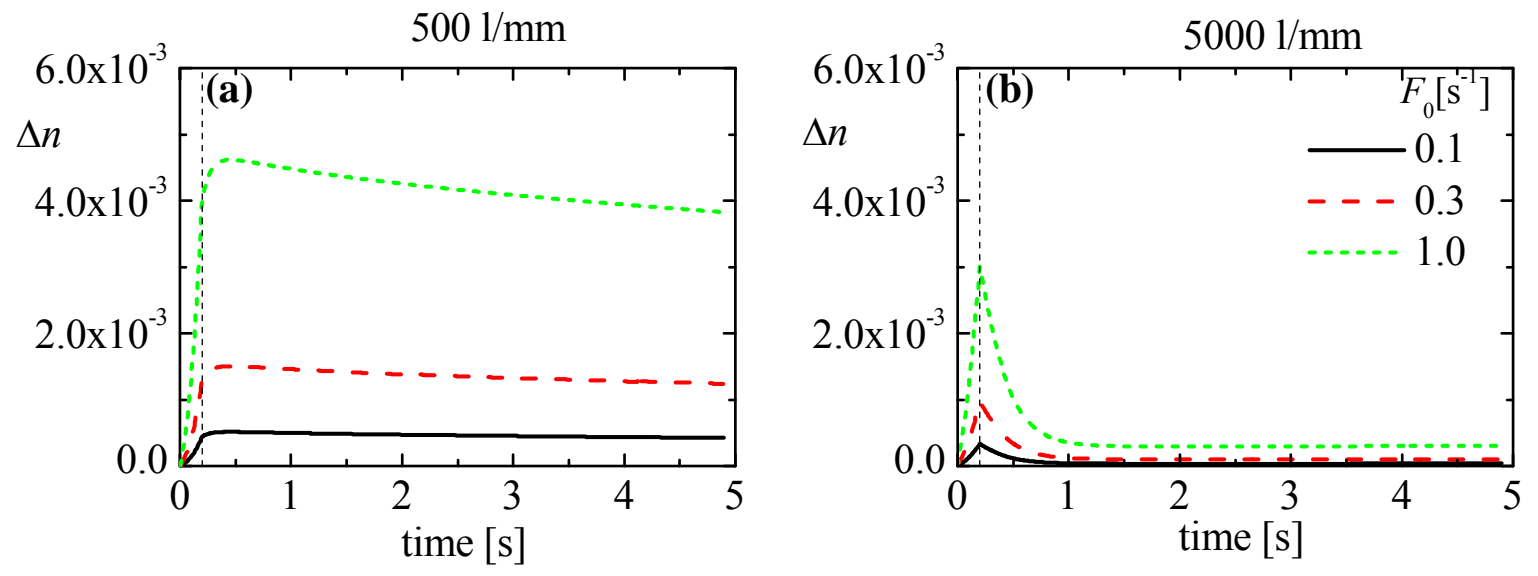

Figure 4 


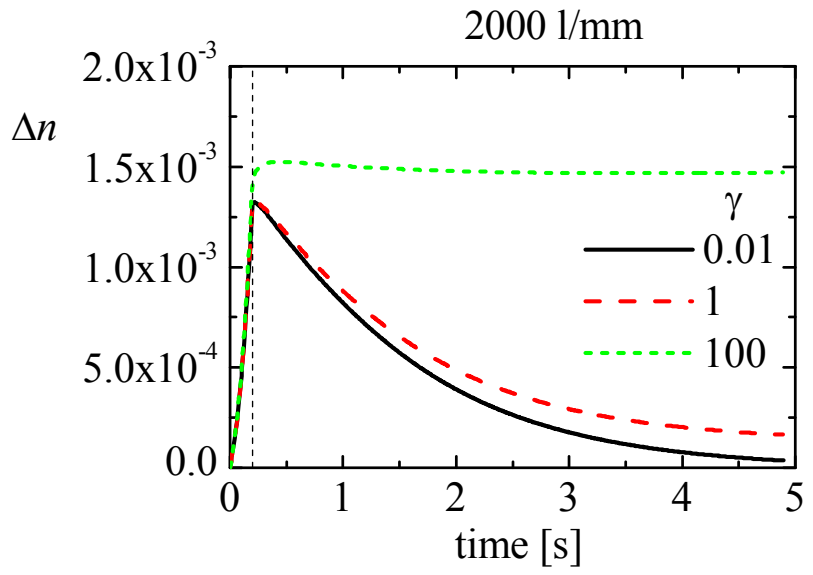

Figure 5 

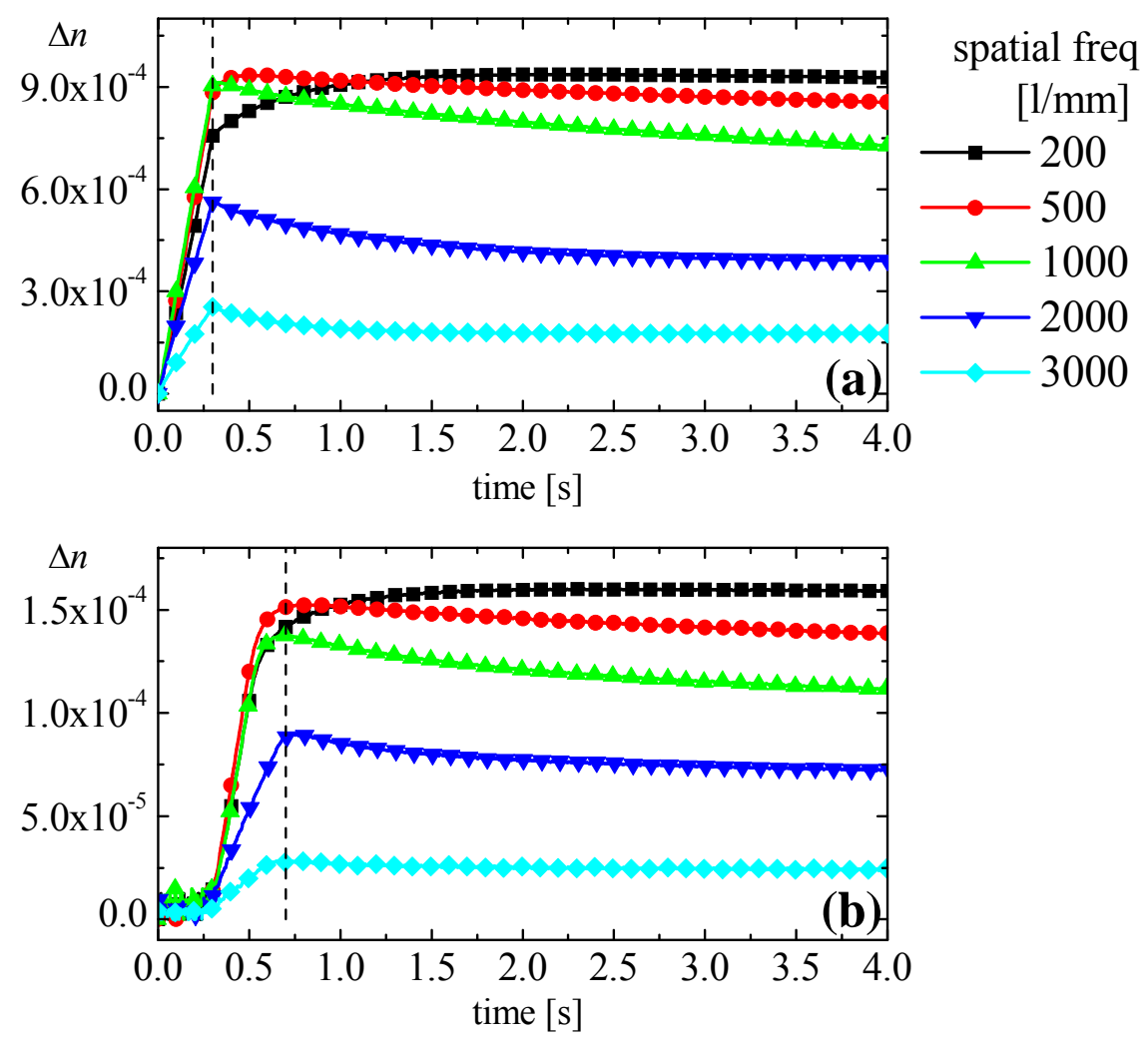

Figure 6 

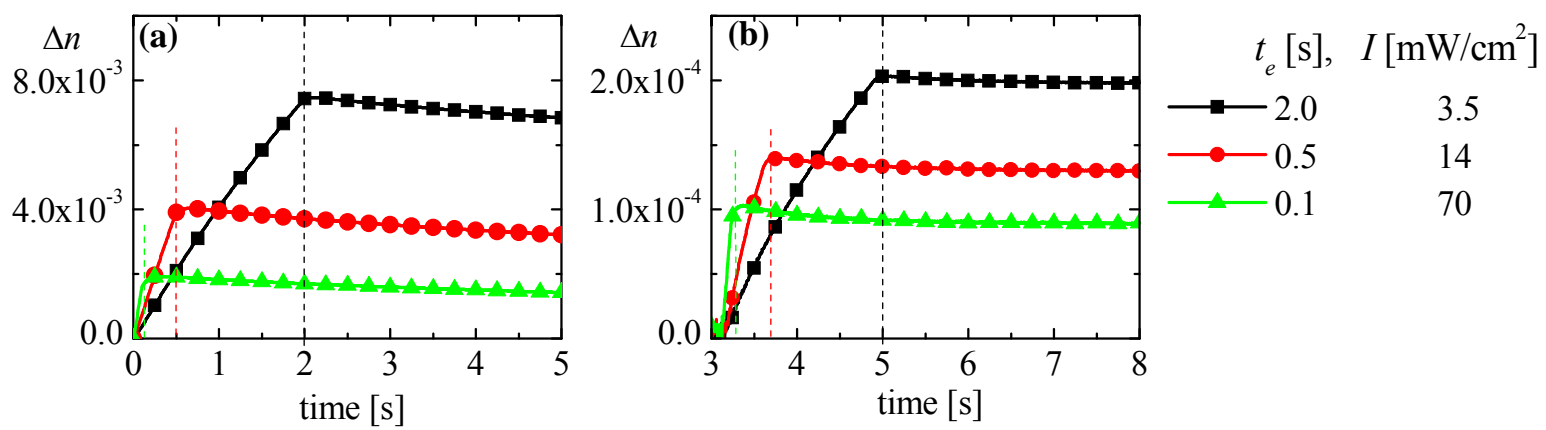

Figure 7 